\title{
A multiwavelength study of filamentary cloud G341.244-00.265
}

\author{
Nai-Ping Yu, Jing-Long Xu, and Jun-Jie Wang
}

\begin{abstract}
National Astronomical Observatories, Chinese Academy of Sciences, Beijing 100012, PR China
\end{abstract}
e-mail: npyu@bao.ac.cn

Received 6 March 2018 / Accepted 29 December 2018

\begin{abstract}
We present a multiwavelength study toward the filamentary molecular cloud G341.244-00.265, to investigate the physical and chemical properties, as well as star formation activities taking place therein. Our radio continuum and molecular line data were obtained from the Sydney University Molonglo Sky Survey (SUMSS), Atacama Pathfinder Experiment Telescope Large Area Survey of the Galaxy (ATLASGAL), Structure, excitation, and dynamics of the inner Galactic interstellar medium (SEDIGISM) and Millimeter Astronomy Legacy Team Survey at $90 \mathrm{GHz}$ (MALT90). The infrared archival data come from Galactic Legacy Infrared Midplane Survey Extraordinaire (GLIMPSE), Wide-field Infrared Survey Explorer (WISE), and Herschel InfraRed Galactic Plane Survey (Hi-GAL). G341.244-00.265 displays an elongated filamentary structure both in far-infrared and molecular line emissions; the "head" and "tail" of this molecular cloud are associated with known infrared bubbles S21, S22, and S24. We made $\mathrm{H}_{2}$ column density and dust temperature maps of this region by the spectral energy distribution (SED) method. G341.244-00.265 has a linear mass density of about $1654 M_{\odot} \mathrm{pc}^{-1}$ and has a projected length of $11.1 \mathrm{pc}$. The cloud is prone to collapse based on the virial analysis. Even though the interactions between this filamentary cloud and its surrounding bubbles are evident, we found these bubbles are too young to trigger the next generation of star formation in G341.244-00.265. From the ATLASGAL catalog, we found eight dense massive clumps associated with this filamentary cloud. All of these clumps have sufficient mass to form massive stars. Using data from the GLIMPSE and WISE survey, we search the young stellar objects (YSOs) in G341.244-00.265. We found an age gradient of star formation in this filamentary cloud: most of the YSOs distributed in the center are Class I sources, while most Class II candidates are located in the head and tail of G341.244-00.265, indicating star formation at the two ends of this filament is prior to the center. The abundance ratio of $\mathrm{N}\left(\mathrm{N}_{2} \mathrm{H}^{+}\right) / \mathrm{N}\left(\mathrm{C}^{18} \mathrm{O}\right)$ is higher in the center than that in the two ends, also indicating that the gas in the center is less evolved. Taking into account the distributions of YSOs and the $\mathrm{N}\left(\mathrm{N}_{2} \mathrm{H}^{+}\right) / \mathrm{N}\left(\mathrm{C}^{18} \mathrm{O}\right)$ ratio map, our study is in agreement with the prediction of the so-called "end-dominated collapse" star formation scenario.
\end{abstract}

Key words. stars: formation - ISM: clouds - ISM: abundances - stars: protostars

\section{Introduction}

Massive stars play an important role in the evolution of our universe. These stars release large amounts of energy into their surrounding interstellar medium (ISM) and have an immense impact on subsequent star formation therein. However, their formation is still poorly understood compared with their lowmass counterparts. One reason is that they are rare and evolve quickly. The other reason is that they used to form in dense clusters in giant molecular clouds (GMCs) at large distances, which makes it hard to study these stars individually. In the last few decades, a lot of research have been done to understand the formation of massive stars (e.g., Zinnecher \& Yorke 2007; Deharveng et al. 2010, and references therein). Based on farinfrared and/or millimeter sky surveys such as Herschel InfraRed Galactic Plane Survey (Hi-GAL; Molinari et al. 2010) and Atacama Pathfinder Experiment Telescope Large Area Survey of the Galaxy (ATLASGAL; (Schuller et al. 2009), it is demonstrated that filamentary structures are ubiquitous in the ISM and play an important role in the processes of star formation. They could fragment into clumps because of gravitational instabilities (e.g., André et al. 2010; Ragan et al. 2014; Li et al. 2016; Dewangan et al. 2017, and references therein). However, the nature of filamentary structures is still unknown. The mechanisms leading to their formation and their link to star formation processes are also still not clear. For a deep understanding of the properties of filamentary molecular clouds, multiwavelength observations are essential.

In order to study the properties of filamentary structures, $\mathrm{Li}$ et al. (2016) recently identified 517 filamentary structure candidates from the ATLASGAL survey. Their study reveals that filaments make a significant contribution to massive star formation. G341.244-00.265 is one of their candidates. According to the work of Schuller et al. (2017), the distance of this filament is about $3.6 \mathrm{kpc}$ to the solar system. Figure 1 shows the Spitzer two-color image of G341.244-00.265. We divide this filamentary structure into three parts: head, body and tail. The head of this filamentary cloud involves three dense massive clumps. The gas distribution shows a shell-like structure around the infrared bubble S24. The interactions between the head and S24 were studied by Cappa et al. (2016). They found that even though star formations are active in this region, the bubble S24 seems too young for triggering to have begun. The body of G341.244-00.265 involves four dense clumps. Two of these (AGAL341.219-00.259 and AGAL341.230-00.271) have been associated with extended green objects (EGO) by Cyganowski et al. (2008). Molecular line observations support that EGOs are good candidates of massive YSOs with ongoing outflow activities (e.g., Chen et al. 2010; Cyganowski et al. 2011). The tail of G341.244-00.265 is associated with S21 and S22. On the border of S22, the dense gas shows an arc-like morphology, indicating that it is also being compressed by the expanding HII region. Toward the south and 


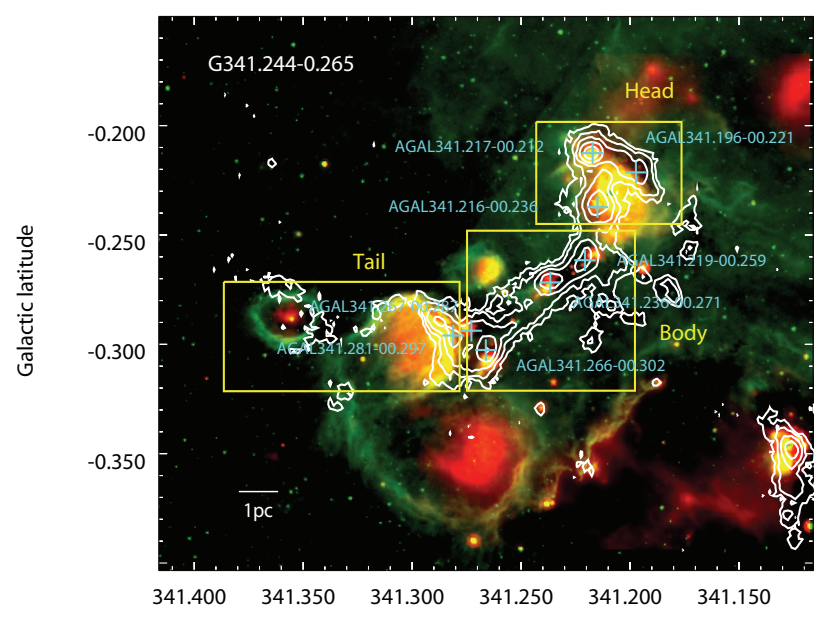

Galactic lonqitude

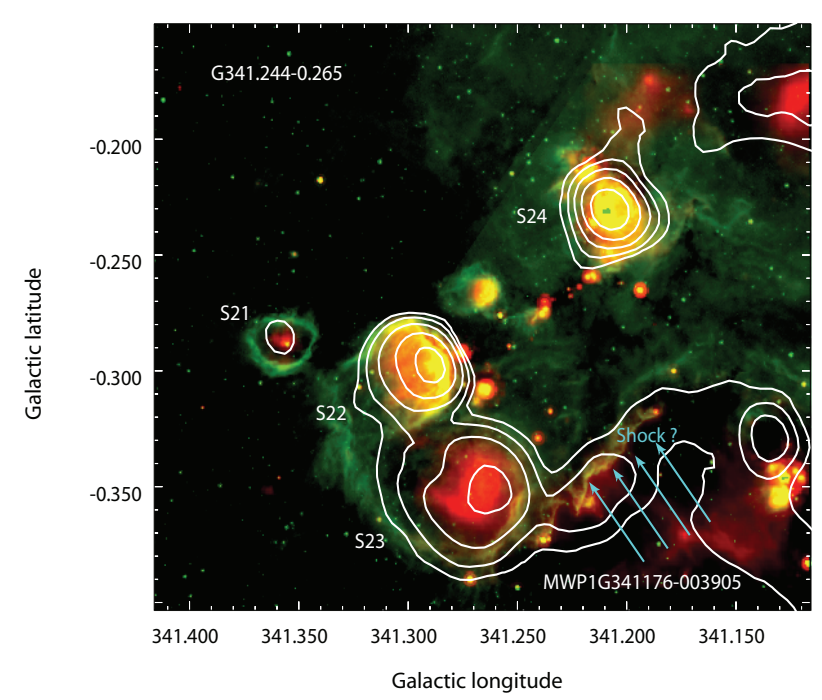

Fig. 1. Two-color image of G341.244-00.265: $8 \mu \mathrm{m}$ emission in green and $24 \mu \mathrm{m}$ emission in red. The magenta plus signs indicate the eight dense clumps from Contreras et al. (2013). The ATLASGAL $870 \mu \mathrm{m}$ emissions (in white) are superimposed with levels 0.2, 0.4, 0.8, 1.6, and $3.2 \mathrm{Jy} \mathrm{beam}^{-1}$ in the top panel. The $843 \mathrm{MHz}$ SUMSS radio continuum emissions (in white) are superimposed with levels 0.02, 0.04, 0.08, 0.16, and $0.32 \mathrm{Jy} \mathrm{beam}^{-1}$ in the bottom panel.

southwest lie bubble S23 and MWP1G341176-003905 (Simpson et al. 2012). The bottom panel of Fig. 1 shows the radio continuum emission at $843 \mathrm{MHz}$ from the Sydney University Molonglo Sky Survey (SUMSS; Mauch et al. 2003). Radio emissions from infrared bubbles S21, S22, S23, S24, and MWP1G341176003905 can be seen. The morphology of radio emission is relatively consistent with that of Spitzer $24 \mu \mathrm{m}$ infrared emissions, which trace warm gas. In order to investigate its physical and chemical properties, as well as star formation activities, we present a multiwavelength study toward this region. We introduce the data we use and analyze in Sect. 2, discussions are given in Sect. 3, and finally we summarize in Sect. 4.

\section{Data and analysis}

In the following, we present our analysis of archival multiwavelength data from Hi-GAL in Sect. 2.1, ATLASGAL in Sect. 2.2, SEDIGISM in Sect. 2.3, and MALT90 in Sect. 2.4.

\section{1. $H i-G A L$}

The Hi-GAL data set is comprised of five continuum images of the Milky Way Galaxy using the PACS (70 and $160 \mu \mathrm{m})$ and SPIRE $(250,350$ and $500 \mu \mathrm{m})$ instruments. This data set helped us identify an unbiased catalog of filament candidates throughout the Galaxy (e.g., Molinari et al. 2010; André et al. 2010; Wang et al. 2015). The angular resolutions range from 5.2" to $35.2^{\prime \prime}$ for 70 and $500 \mu \mathrm{m}$, respectively. The high-frequency bands provide high angular resolution and are less affected by large-scale background and foreground emissions. We made $\mathrm{H}_{2}$ column density and dust temperature maps of this region by the spectral energy distribution (SED) method described by Wang et al. (2015). Given Hi-GAL is sensitive to low-density gas of about $10^{21} \mathrm{~cm}^{-2}$, background and/or foreground contaminations create a serious problem when analyzing the Hi-GAL data. Following the steps described by Wang et al. (2015), we first removed the background and foreground emissions. After removing the background and foreground emissions, we convolved all images at a resolution of $35^{\prime \prime}$, which is the beam size of Hi-GAL at $500 \mu \mathrm{m}$. For each pixel, we used equation

$I_{v}=B_{v}\left(1-e^{-\tau_{v}}\right)$

to model intensities at various wavelengths. The optical depth $\tau_{v}$ could be estimated through

$\tau_{v}=\mu_{\mathrm{H}_{2}} m_{\mathrm{H}} \kappa_{v} N_{\mathrm{H}_{2}} / R$.

We adopted a mean molecular weight per $\mathrm{H}_{2}$ molecule of $\mu_{\mathrm{H}_{2}}=2.8$ to include the contributions from helium and other heavy elements. The value $m_{\mathrm{H}}$ is the mass of a hydrogen atom, $N_{\mathrm{H}_{2}}$ is the column density, and $R$ is the gas-to-dust mass ratio, which is set to be 100 . According to Ossenkopf \& Henning (1994), dust opacity per unit dust mass $\left(\kappa_{v}\right)$ could be expressed as

$\kappa_{v}=5.0\left(\frac{v}{600 \mathrm{GHz}}\right)^{\beta} \mathrm{cm}^{-2} \mathrm{~g}^{-1}$,

where the value of the dust emissivity index $\beta$ is fixed to 1.75 in our fitting. The two free parameters $\left(N_{\mathrm{H}_{2}}\right.$ and $\left.T_{d}\right)$ for each pixel could be fitted finally. Figure 2 shows the derived $\mathrm{H}_{2}$ column density and dust temperature maps of G341.24400.265 . The morphology of column density is similar to the ATLASGAL $870 \mu \mathrm{m}$ emissions. The mean $\mathrm{H}_{2}$ column density is $1.9 \times 10^{22} \mathrm{~cm}^{-2}$. The total mass of G341.244-00.265 is $\sim 18919$ $M_{\odot}$ and has a projected length of $11.1 \mathrm{pc}$. In the head and tail, the gas is much warmer than that in the body of G341.24400.265 . This is probably because the dust there is heated by S22 and S24.

\subsection{ATLASGAL clumps}

The ATLASGAL also helped us identify an unbiased catalog of filament candidates throughout the Galaxy in the emissions of $870 \mu \mathrm{m}$ ( $\mathrm{Li}$ et al. 2016). This is the first systematic survey of the inner Galactic plane in the submillimeter (Siringo et al. 2009; Contreras et al. 2013). It provides high angular resolution $(\sim 19.2 \mathrm{arcsec})$ of cold dust emissions in the Galaxy. For a dust temperature of $20 \mathrm{~K}$, ATLASGAL is sensitive to gas with $\mathrm{H}_{2}$ column densities exceeding $10^{22} \mathrm{~cm}^{-2}$. We found eight dense ATLASGAL clumps from the catalog of Contreras et al. (2013) distributing along this filament like beads on a string. From Table 1, we can see that most of these clumps have masses 

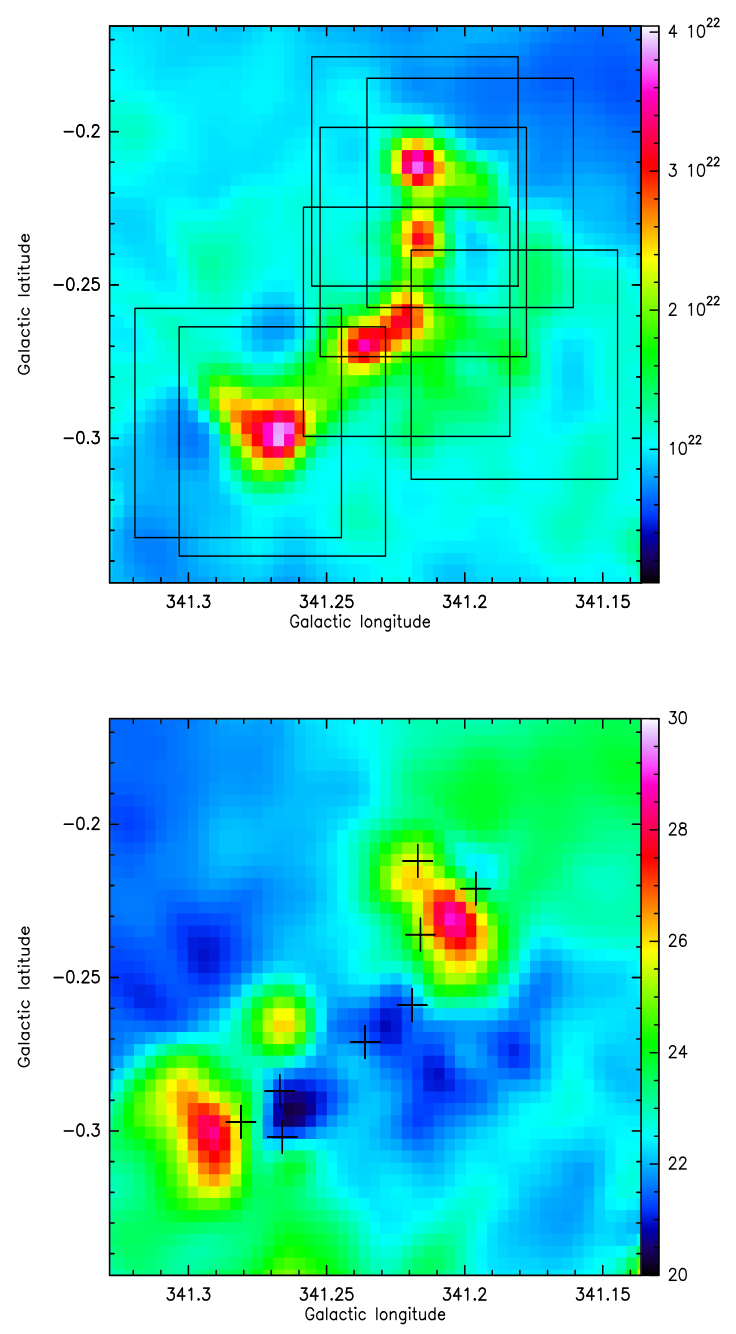

Fig. 2. Top panel: $\mathrm{H}_{2}$ column density map of G341.244-00.265 built on the SED fitting pixel by pixel. The seven boxes indicate the seven observed regions by MALT90. Bottom panel: dust temperature $T_{\text {dust }}$ map of G341.244-00.265. The plus signs indicate the dense clumps from Contreras et al. (2013).

Table 1. Physical parameters of the ATLASGAL clumps.

\begin{tabular}{ccccc}
\hline \hline $\begin{array}{c}\text { Source } \\
\text { name }\end{array}$ & $\begin{array}{c}l \\
(\mathrm{deg})\end{array}$ & $\begin{array}{c}b \\
(\mathrm{deg})\end{array}$ & $\begin{array}{c}R_{\mathrm{eff}}^{a} \\
(\mathrm{pc})\end{array}$ & $\begin{array}{c}\mathrm{M}^{a} \\
\left(M_{\odot}\right)\end{array}$ \\
\hline AGAL341.196-00.221 & 341.196 & -0.221 & 0.42 & 837 \\
AGAL341.217-00.212 & 341.217 & -0.212 & 0.46 & 1070 \\
AGAL341.216-00.236 & 341.216 & -0.236 & 0.83 & 1390 \\
AGAL341.219-00.259 & 341.219 & -0.259 & 0.75 & 1910 \\
AGAL341.236-00.271 & 341.236 & -0.271 & 0.57 & 1290 \\
AGAL341.266-00.302 & 341.266 & -0.302 & 0.70 & 2290 \\
AGAL341.281-00.297 & 341.281 & -0.297 & 0.68 & 1320 \\
AGAL341.267-00.287 & 341.267 & -0.287 & 0.53 & 360 \\
\hline
\end{tabular}

Notes. ${ }^{(a)}$ These values are from Contreras et al. (2017).

$>10^{3} M_{\odot}$. Given a typical star formation efficiency of $10-30 \%$ Lada et al. (2010) and a cluster having a Salpeter-type initial stellar mass function (IMF), we could expect a $10^{3} M_{\odot}$ clump to form a star cluster with massive stars $>20 M_{\odot}$. Therefore, this filamentary cloud is a candidate of massive star-forming region.

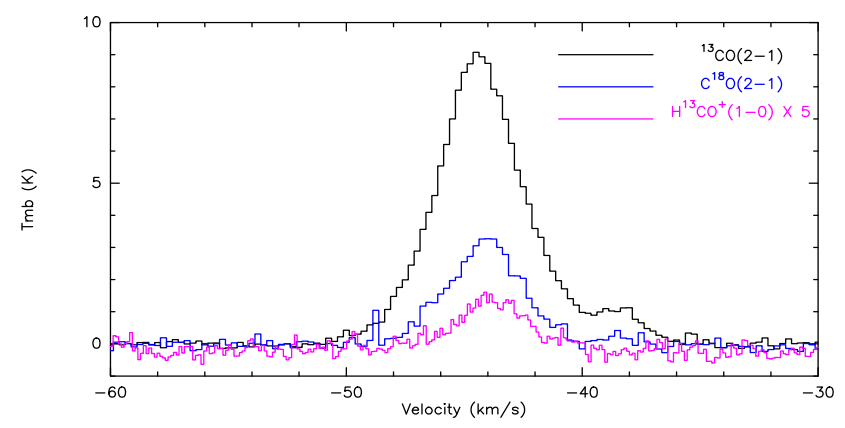

Fig. 3. Averaged spectra of ${ }^{13} \mathrm{CO}(2-1), \mathrm{C}^{18} \mathrm{O}(2-1)$ and $\mathrm{H}^{13} \mathrm{CO}^{+}(1-0)$ over the filamentary molecular cloud of G341.244-00.265.

\subsection{SEDIGISM}

We analyzed ${ }^{13} \mathrm{CO}(2-1)$ and $\mathrm{C}^{18} \mathrm{O}(2-1)$ emissions in the region of G341.244-00.265 using the Structure, excitation, and dynamics of the inner Galactic interstellar medium (SEDIGISM; Schuller et al. 2017) data. This survey used the lowest frequency module of the Swedish Heterodyne Facility Instrument (SHFI; Vassilev et al. 2008) arrayed on the $12 \mathrm{~m}$ Atacama Pathfinder Experiment telescope (APEX), which is located on Llano de Chajnantor in Chile. This project covers a longitude in the range $l=-60^{\circ}$ to $18^{\circ}$ and a latitude in the range $|b|<0.5^{\circ}$ and has a beam size of $28^{\prime \prime}$. The $4 \mathrm{GHz}$ bandwidth spectrometer consists of two backend units, Fast Fourier Transform Spectrometer-1 (XFFTS-1; Klein et al. 2012) and XFFTS-2. The bandwidth of each unit is $2.5 \mathrm{GHz}$ and has an overlap of $1000 \mathrm{MHz}$. The velocity resolution is about $0.1 \mathrm{~km} \mathrm{~s}^{-1}$ at the frequency near $220 \mathrm{GHz}$. In addition to the $\mathrm{CO}$-isotopolog lines, this survey also includes other transitions such as $\mathrm{SO}(5-4), \mathrm{SiO}(5-4), \mathrm{HC}_{3} \mathrm{~N}(24-23)$, and so on. According to the APEX telescope efficiencies home page $^{1}$, the beam efficiency of APEX is 0.75 at the frequency of $230 \mathrm{GHz}$. A more detail introduction of this survey can be found in Schuller et al. (2017). The ${ }^{13} \mathrm{CO}(2-1)$ and $\mathrm{C}^{18} \mathrm{O}(2-1)$ cubes are publicly available and can be downloaded from a dedicated server hosted by MPIfR ${ }^{2}$. Using software packages of CLASS (Continuum and Line Analysis Single-Disk Software) and GREG (Grenoble Graphic), we conducted the data analysis. To compare with the reduced data of Hi-GAL introduced above and the following data of MALT90, a Gaussian smoothing was applied to convolve the SEDIGISM data into a new resolution of $38^{\prime \prime}$.

Figure 3 shows the averaged spectra of ${ }^{13} \mathrm{CO}(2-1)$ and $\mathrm{C}^{18} \mathrm{O}(2-1)$ over the filamentary molecular cloud of G341.24400.265 . It can be seen that two components at velocity intervals -48 to $-40 \mathrm{~km} \mathrm{~s}^{-1}$ and -40 to $-36 \mathrm{~km} \mathrm{~s}^{-1}$ are detected by the two $\mathrm{CO}$ isotopologs. The former component is consistent with the ranges measured by Schuller et al. (2017). On the other hand, the -40 to $-36 \mathrm{~km} \mathrm{~s}^{-1}$ component is undetected by $\mathrm{H}^{13} \mathrm{CO}^{+}(1-0)$ in the MALT90 data. Thus, it is likely to be foreground or background emissions unrelated with G341.24400.265 . We made the integrated intensity maps of ${ }^{13} \mathrm{CO}(2-1)$ and $\mathrm{C}^{18} \mathrm{O}(2-1)$ in the velocity range -48 to $-40 \mathrm{~km} \mathrm{~s}^{-1}$. The emission contours overlaid on the Spitzer-IRAC $8.0 \mu \mathrm{m}, \mathrm{H}_{2}$ column density, and dust temperature maps derived in Sect. 2.1 are shown in Fig. 4. The regions traced by ${ }^{13} \mathrm{CO}(2-1)$ show an elongated filamentary structure extending from northwest to

\footnotetext{
1 http://www.apex-telescope.org/telescope/efficiency/ index.php

2 http://sedigism.mpifr-bonn.mpg.de
} 

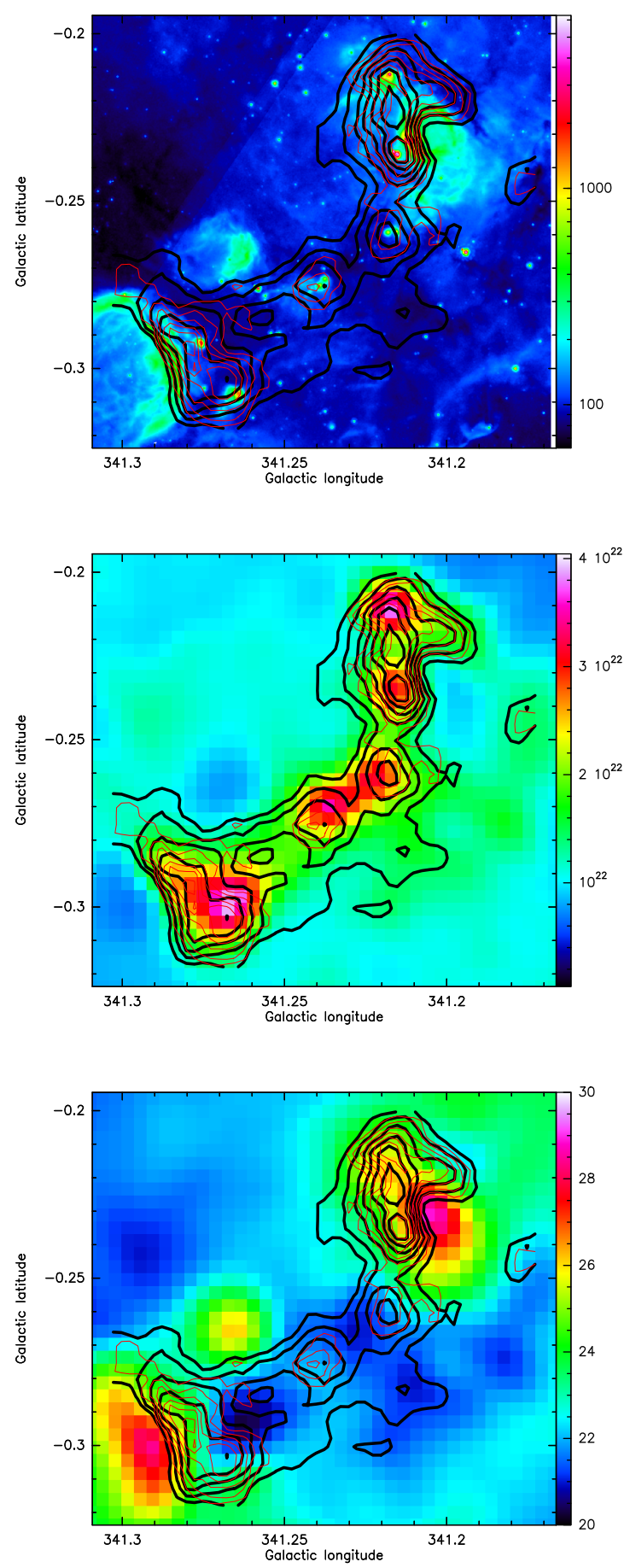

Fig. 4. ${ }^{13} \mathrm{CO}$ (black) and $\mathrm{C}^{18} \mathrm{O}$ (red) line emission contours superimposed on the images of Siptzer-IRAC $8.0 \mu \mathrm{m}$ (top panel), $\mathrm{H}_{2}$ column density (middle panel), and dust temperature (bottom panel). The emissions are integrated from -48 to $-40 \mathrm{~km} \mathrm{~s}^{-1}$. Contour levels are 40 , $50, \ldots, 90 \%$ of each peak emission.

southeast. On the borders of S22 and S24, a good morphological match can be seen between photodissociation regions (PDRs) and $\mathrm{CO}$ emissions, indicating the gas is being compressed by the two infrared bubbles. The $\mathrm{C}^{18} \mathrm{O}(2-1)$ emission traces more compact gas than the ${ }^{13} \mathrm{CO}(2-1)$ emission owing to its lower abundance. Most of the $\mathrm{C}^{18} \mathrm{O}$ emissions come from the head and tail of G341.244-00.265, where the dust temperature is relatively high. In the body, where the dust temperature is around $20 \mathrm{~K}$, the $\mathrm{C}^{18} \mathrm{O}$ emission is relatively weak. Chemical models indicate that when $T_{d}$ is below $20 \mathrm{~K}$, carbon species like $\mathrm{CO}$ and $\mathrm{CS}$ can be depleted in the cold gas (e.g., Lee et al. 2003; Bergin \& Tafalla 2007). This may be the reason that the $\mathrm{C}^{18} \mathrm{O}$ emission is relatively weak in the body of G341.244-00.265.

The $\mathrm{C}^{18} \mathrm{O}$ line is very useful to quantify the column density, as its emissions are optically thin in most cases. We used the $\mathrm{C}^{18} \mathrm{O}(2-1)$ to derive the column density by assuming the local thermodynamic equilibrium (LTE) conditions. The optical depth of $\mathrm{C}^{18} \mathrm{O}(2-1)$ can be estimated by comparing with its isotopolog line ${ }^{13} \mathrm{CO}(2-1)$. We assumed that the ${ }^{13} \mathrm{CO}$ and $\mathrm{C}^{18} \mathrm{O}$ emissions arise from the same gas and share a common excitation temperature. The optical depth of the $\mathrm{C}^{18} \mathrm{O}$ line could be derived through

$\frac{T_{\mathrm{mb}}\left({ }^{13} \mathrm{CO}\right)}{T_{\mathrm{mb}}\left(\mathrm{C}^{18} \mathrm{O}\right)}=\frac{1-e^{-A \tau_{18}}}{1-e^{-\tau_{18}}}$

where $A$ is their isotope abundance ratio. In this paper, we adopted the isotopic ratio from Wilson \& Rood (1994), which depends on the Galactocentric radius of the region,

$A \equiv \frac{\left[{ }^{13} \mathrm{CO}\right]}{\left[\mathrm{C}^{18} \mathrm{O}\right]} \sim \frac{\left[{ }^{13} \mathrm{C}\right]\left[{ }^{16} \mathrm{O}\right]}{\left[{ }^{12} \mathrm{C}\right]\left[{ }^{18} \mathrm{O}\right]}=\frac{58.8 \times R_{\mathrm{GC}}[\mathrm{kpc}]+37.1}{7.5 \times R_{\mathrm{GC}}[\mathrm{kpc}]+7.6}$,

where $R_{\mathrm{GC}}$ is the distance of the molecular cloud to the Galactic center. By solving Eq. (4) in every map pixel, we obtained the map of $\tau_{18}$, which is shown in the top panel of Fig. 5. The derived $\tau_{18}$ is in the range 0.2 and 0.5 , indicating the $\mathrm{C}^{18} \mathrm{O}$ emission is indeed optically thin in this filamentary cloud.

Assuming a filled telescope beam and the excitation temperature is the same for the two isotopic species, we calculated the excitation temperature of $\mathrm{C}^{18} \mathrm{O}$ using

$T_{\mathrm{ex}}=\frac{h v_{0}}{k}\left[\ln \left(1+\frac{h v_{0} / k}{T_{\mathrm{mb}} /\left(1-e^{-\tau}\right)+J_{v}\left(T_{\mathrm{bg}}\right)}\right)\right]^{-1}$,

where $v_{0}$ is the rest frequency of the transition, $T_{\mathrm{bg}}$ is the temperature of the background radiation $(2.73 \mathrm{~K})$, and

$J_{\nu}(T)=\frac{h v_{0}}{k} \frac{1}{e^{h v_{0} / k T}-1}$.

Once $\tau_{18}$ and $T_{\text {ex }}$ are obtained, the column density of $\mathrm{C}^{18} \mathrm{O}$ can be calculated through equation

$N\left(\mathrm{C}^{18} \mathrm{O}\right)=\frac{8 \pi v_{0}^{3}}{c^{3}(2 J+1) A} \sqrt{1+\left(\frac{2 J T_{\mathrm{ex}}}{T_{0}}\right)^{2}} \frac{e^{(J+1) T_{0} / 2 T_{\mathrm{ex}}}}{e^{T_{0} / T_{\mathrm{ex}}}-1} \int \tau_{18} \mathrm{~d} v$,

where $\mathrm{A}$ is the Einstein coefficient for spontaneous transition, $T_{0} \equiv h v_{0} / k=10.55 \mathrm{~K}$ for $\mathrm{C}^{18} \mathrm{O}(2-1)$. On the other hand, given $\mathrm{C}^{18} \mathrm{O}(2-1)$ is optically thin, we used the approximation

$\int \tau_{18} \mathrm{~d} v=\frac{1}{J\left(T_{\mathrm{ex}}\right)-J\left(T_{\mathrm{BG}}\right)} \int T_{\mathrm{mb}} \mathrm{d} v$

The abundance of $\mathrm{C}^{18} \mathrm{O}$ in each pixel can finally be calculated through $\chi\left(\mathrm{C}^{18} \mathrm{O}\right)=\mathrm{N}\left(\mathrm{C}^{18} \mathrm{O}\right) / \mathrm{N}\left(\mathrm{H}_{2}\right)$. The column density and abundance maps of $\mathrm{C}^{18} \mathrm{O}$ are also shown in Fig. 5. The obtained $\mathrm{C}^{18} \mathrm{O}$ abundance is $(1.9-29.9) \times 10^{-7}$ and has a mean value of $8.9 \times 10^{-7}$. It can be noted that the $\mathrm{C}^{18} \mathrm{O}$ column density is relatively high on the PDRs around S22 and S24, where the gas is relatively warm. The $\mathrm{T}_{d}-\chi\left(\mathrm{C}^{18} \mathrm{O}\right)$ relation map in Fig. 6 suggests a positive correlation. This is consistent with chemical model results that as gas gets warmer, more $\mathrm{CO}$ species are evaporated from dust grains. 

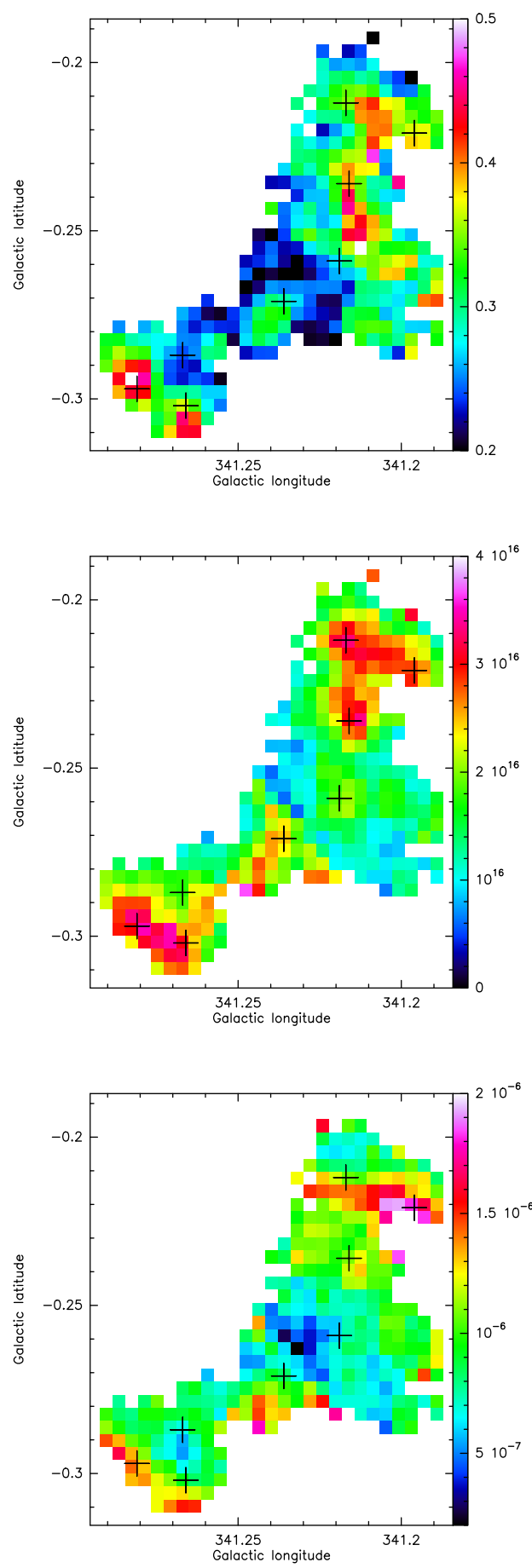

Fig. 5. Maps of the optical depth (top panel), column density (middle panel), and abundance (bottom panel) of $\mathrm{C}^{18} \mathrm{O}$. The plus signs indicate the dense clumps from Contreras et al. (2013).

\subsection{MALT9O}

The $\mathrm{N}_{2} \mathrm{H}^{+}$molecular line data are from MALT90. It is one of the most detected molecules in this survey (e.g., Rathborne 2016). MALT90 is an international project aimed at characterizing the sites within our Galaxy where massive star formation take place (e.g., Foster et al. 2011; Jackson et al. 2013). This project was carried out with the Mopra Spectrometer (MOPS) mounted on the Mopra $22 \mathrm{~m}$ telescope, which is located near Coonabarabran in New South Wales, Australia. The full $8 \mathrm{GHz}$ bandwidth of MOPS was split into 16 zoom bands of $138 \mathrm{MHz}$, providing a

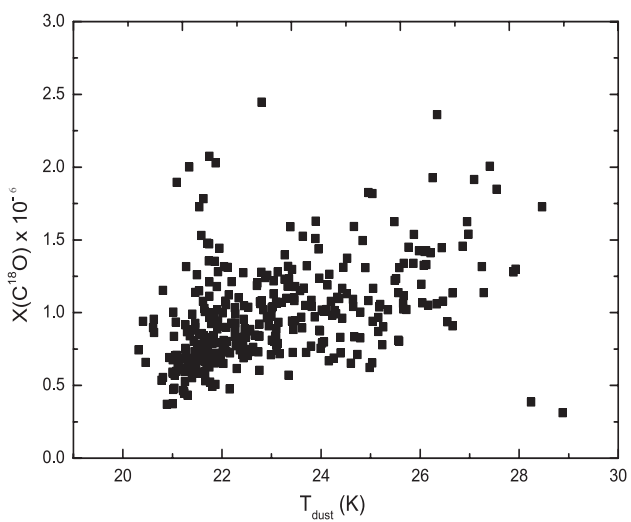

Fig. 6. Abundance of $\mathrm{C}^{18} \mathrm{O}$ plotted as a function of dust temperature in each pixel of G341.244-00.265.

velocity resolution of $0.11 \mathrm{~km} \mathrm{~s}^{-1}$ at frequencies near $90 \mathrm{GHz}$. The angular resolution of Mopra is $38^{\prime \prime}$ and has a beam efficiency between 0.49 at $86 \mathrm{GHz}$ and 0.42 at $115 \mathrm{GHz}$ (Ladd et al. 2005). The targets of this survey are selected from the ATLASGAL clumps found by Contreras et al. (2013). The size of the data cube is $4.6^{\prime} \times 4.6^{\prime}$ and has a step of $9^{\prime \prime}$. The data files are publicly available and can be downloaded from the MALT90 home page ${ }^{3}$. We searched the region of G341.244-00.265 and found seven dust clumps from Contreras et al. (2013) that have been observed by MALT90. The seven regions observed by MALT90 are shown in Fig. 2. We combined the seven data sets into a new data cube by CLASS, keeping the same beam size and spacing between adjacent rows. The new combined image of $\mathrm{N}_{2} \mathrm{H}^{+}$is shown in Fig. 7.

$\mathrm{N}_{2} \mathrm{H}^{+}$is a good tracer of dense gas in the early stages of star formation as it is more resistant to freeze-out on grains than the carbon-bearing species (Bergin et al. 2001). The emission maps of $\mathrm{N}_{2} \mathrm{H}^{+}$overlaid on the Spitzer-IRAC $8 \mu \mathrm{m}, \mathrm{H}_{2}$ column density, and dust temperature are shown in Fig. 8. We can see that the morphology of $\mathrm{N}_{2} \mathrm{H}^{+}$integrated intensity is very similar to that of the $\mathrm{H}_{2}$ column density. $\mathrm{N}_{2} \mathrm{H}^{+}(1-0)$ has 15 hyperfine transitions of which 7 have a different frequency (e.g., Pagani et al. 2009; Keto \& Rybicki 2010). As shown in Fig. 7, in our filament G341.244-00.265, the 7 hyperfine structures of $\mathrm{N}_{2} \mathrm{H}^{+}$ (1-0) blended into 3 groups because of turbulent line widths. Following the method described by Purcell et al. (2009), we estimate the optical depth of $\mathrm{N}_{2} \mathrm{H}^{+}(1-0)$. Assuming that the line widths of the individual hyperfine components are all equal, the integrated intensities of group 1/group 2 (defined by Purcell et al. 2009) should be in the ratio of 1:5. The optical depth of $\mathrm{N}_{2} \mathrm{H}^{+}\left(\tau_{\mathrm{N}_{2} \mathrm{H}^{+}}\right)$can then be derived using the following equation:

$\frac{\int T_{\mathrm{MB}, 1} \mathrm{~d} v}{\int T_{\mathrm{MB}, 2} \mathrm{~d} v}=\frac{1-\exp \left(-0.2 \tau_{2}\right)}{1-\exp \left(-\tau_{2}\right)}$.

By solving Eq. (10) in every map pixel, we obtained the map of $\tau_{\mathrm{N}_{2} \mathrm{H}^{+}}$, which is shown in the top panel of Fig. 9. We found that in most part of this molecular cloud, the $\mathrm{N}_{2} \mathrm{H}^{+}(1-0)$ has an intermediate optical depth, ranging from 0.2 to 0.8 . Then, we used the following formula to calculate the excitation temperature $\left(T_{\mathrm{ex}}\right)$ of $\mathrm{N}_{2} \mathrm{H}^{+}$:

$T_{\mathrm{ex}}=4.47 / \ln \left(1+\left(\frac{T_{\mathrm{mb}}}{4.47(1-\exp (-\tau))}+0.236\right)^{-1}\right)$.

3 http://atoa.atnf.csiro.au/MALT90 

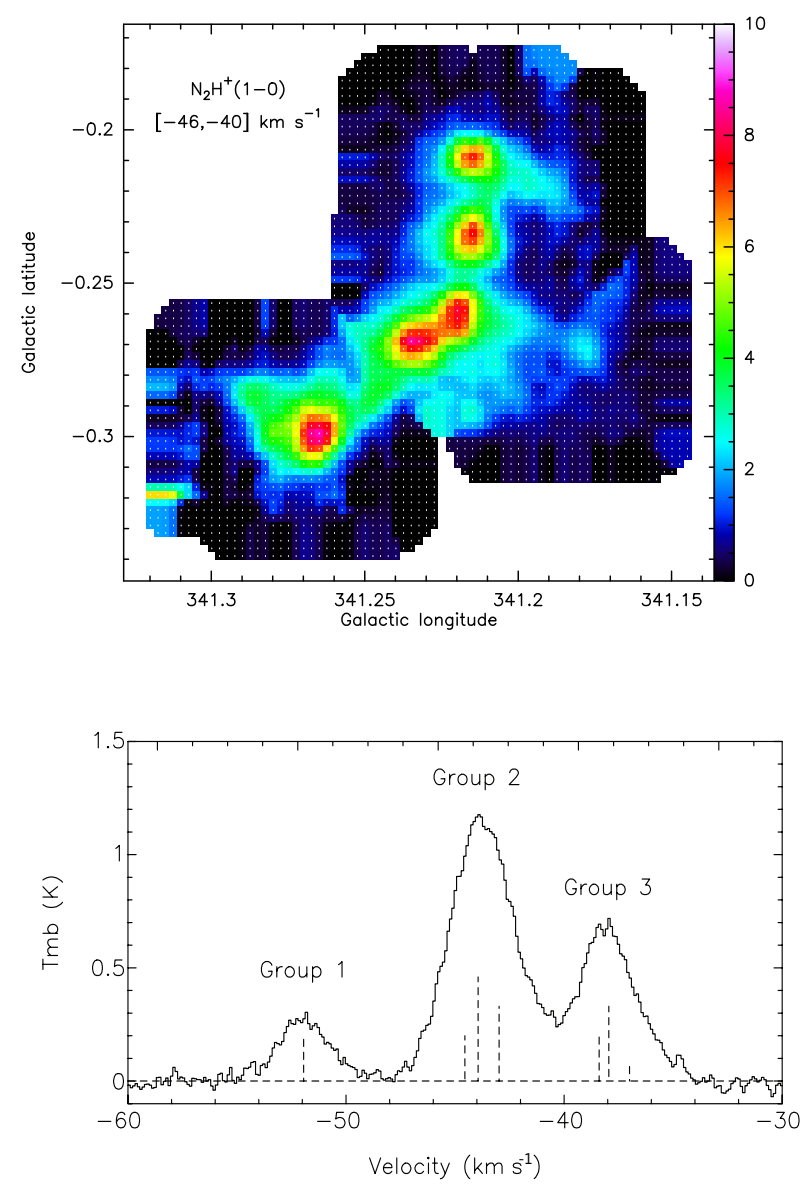

Fig. 7. Top panel: new combined image of $\mathrm{N}_{2} \mathrm{H}^{+}$from MALT90 data set. The emission has been integrated from -46 to $-40 \mathrm{~km} \mathrm{~s}^{-1}$. Bottom panel: averaged spectra of $\mathrm{N}_{2} \mathrm{H}^{+}$over the cloud of G341.244-00.265. The vertical dashed lines indicate the seven hyperfine structures.

Assuming LTE conditions and a beam filling factor of 1, the column density of $\mathrm{N}_{2} \mathrm{H}^{+}$in every pixel can thus be calculated through

$N\left(\mathrm{~N}_{2} \mathrm{H}^{+}\right)=\frac{8 \pi v^{3}}{c^{3} R} \frac{Q_{\mathrm{rot}}}{g_{\mathrm{u}} A_{\mathrm{ul}}} \frac{\exp \left(E_{1} / k T_{\mathrm{ex}}\right)}{1-\exp \left(-h v / k T_{\mathrm{ex}}\right)} \int \tau \mathrm{d} v$,

where $c$ is the velocity of light in the vacuum, $v$ is the frequency of the transition, $g_{\mathrm{u}}$ is the statistical weight of the upper level, $A_{\mathrm{ul}}$ is the Einstein coefficient, $E_{\mathrm{l}}$ is the energy of the lower level, and $Q_{\text {rot }}$ is the partition function. We used the approximation

$\int \tau \mathrm{d} v=\frac{\tau}{1-\exp (-\tau)} \frac{\int T_{\mathrm{mb}} \mathrm{d} v}{J\left(T_{\mathrm{ex}}\right)-J\left(T_{\mathrm{bg}}\right)}$

to take $\tau_{\mathrm{N}_{2} \mathrm{H}^{+}}$into account. The abundance of $\mathrm{N}_{2} \mathrm{H}^{+}$in each pixel can be calculated through $\chi\left(\mathrm{N}_{2} \mathrm{H}^{+}\right)=\mathrm{N}\left(\mathrm{N}_{2} \mathrm{H}^{+}\right) / \mathrm{N}\left(\mathrm{H}_{2}\right)$. The column density and abundance maps of $\mathrm{N}_{2} \mathrm{H}^{+}$is also shown in Fig. 9. In G341.244-00.265, the column density of $\mathrm{N}_{2} \mathrm{H}^{+}$ ranges from $2.1 \times 10^{12}$ to $1.8 \times 10^{13} \mathrm{~cm}^{-2}$ and has a mean value of $0.9 \times 10^{13} \mathrm{~cm}^{-2}$. In the dense part of this molecular cloud, the morphology of $\mathrm{N}_{2} \mathrm{H}^{+}$column density is very similar to that of $\mathrm{N}_{\mathrm{H}_{2}}$, suggesting $\mathrm{N}_{2} \mathrm{H}^{+}$is really a good tracer for dense gas.

Chemical models indicate that in the early stages of star formation, as the cloud collapses and the density increases, $\mathrm{C}$-species including $\mathrm{CO}$ are easy to be absorbed onto the dust
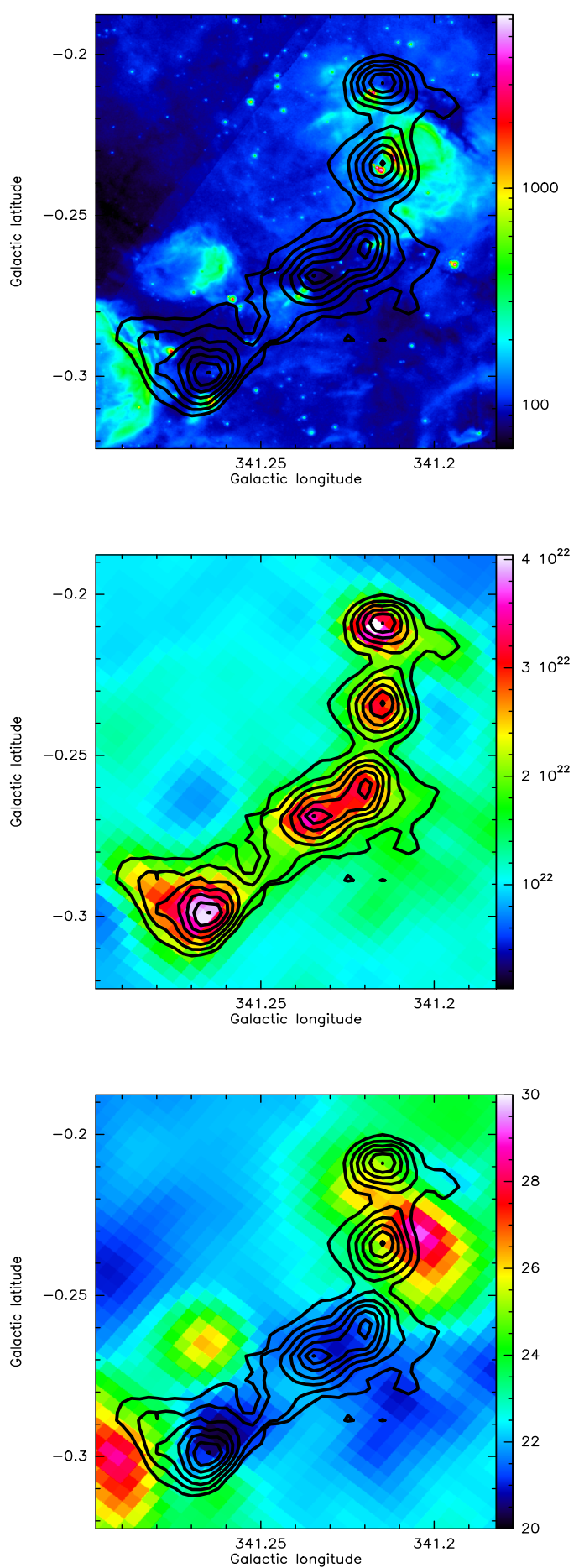

Fig. 8. $\mathrm{N}_{2} \mathrm{H}^{+}$emission contours superimposed on the images of SpitzerIRAC $8.0 \mu \mathrm{m}$ (top panel), $\mathrm{H}_{2}$ column density (middle panel), and dust temperature (bottom panel). Contour levels are from 2.5 to 8.5 in step of $1.0 \mathrm{~K} \mathrm{~km} \mathrm{~s}^{-1}$.

surface, while $\mathrm{N}$-bearing species such as $\mathrm{NH}_{3}$ and $\mathrm{N}_{2} \mathrm{H}^{+}$are hardly depleted. As the central star evolves, the molecular cloud gets warm and $\mathrm{CO}$ is evaporated from the dust grains when the dust temperature exceeds $\sim 20 \mathrm{~K}$ (Tobin et al. 2013). $\mathrm{N}_{2} \mathrm{H}^{+}$ could be destroyed by $\mathrm{CO}$ through $\mathrm{N}_{2} \mathrm{H}^{+}+\mathrm{CO} \longrightarrow \mathrm{HCO}^{+}+\mathrm{N}_{2}$ (e.g., Bergin \& Langer 1997; Lee et al. 2004; Yu \& Xu 2016). When HII regions have formed, $\mathrm{N}_{2} \mathrm{H}^{+}$could also be destroyed by the electron recombination $\mathrm{N}_{2} \mathrm{H}^{+}+\mathrm{e}^{-} \longrightarrow \mathrm{N}_{2}+\mathrm{H}$ or $\mathrm{NH}+\mathrm{N}$ (e.g., Busquet et al. 2011; Dislaire et al. 2012; 

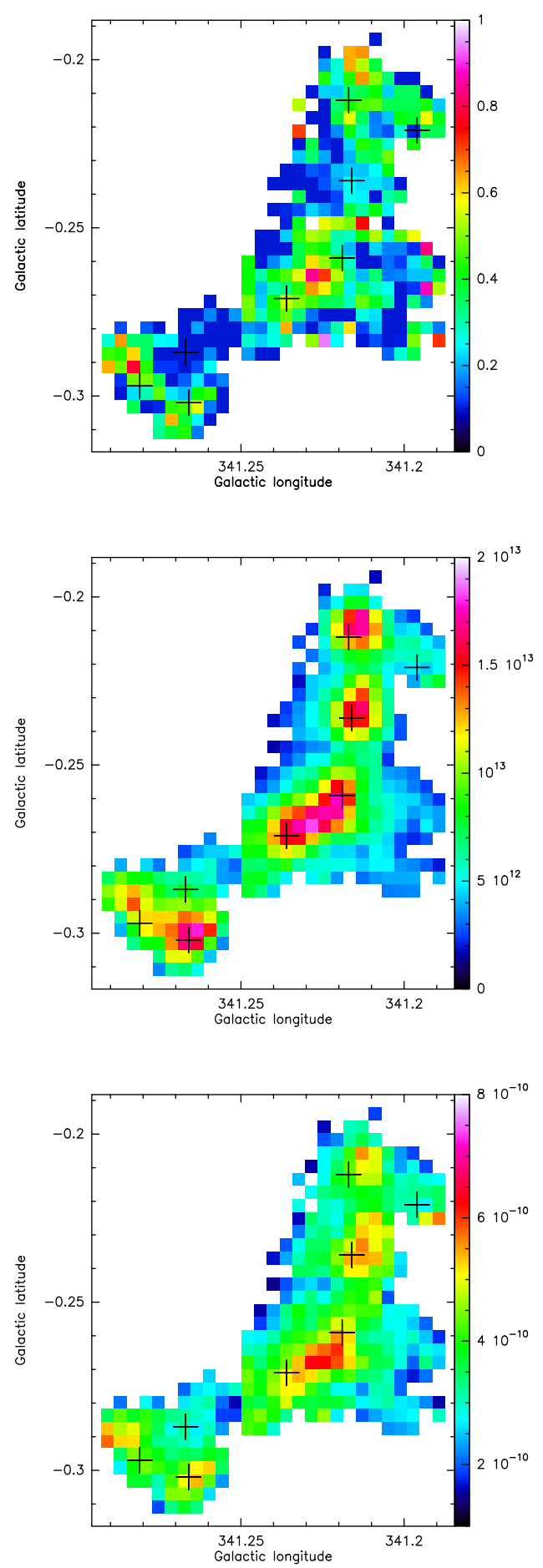

Fig. 9. Maps of the optical depth (top panel), column density (middle panel), and abundance (bottom panel) of $\mathrm{N}_{2} \mathrm{H}^{+}(1-0)$. The plus signs indicate the dense clumps from Contreras et al. (2013).

Vigren et al. 2012; Yu \& $\mathrm{Xu}$ 2016). Thus, the $\mathrm{N}\left(\mathrm{N}_{2} \mathrm{H}^{+}\right) / \mathrm{N}\left(\mathrm{C}^{18} \mathrm{O}\right)$ ratio could be used as a chemical clock for cloud evolution in star-forming regions. We would expect to find that this ratio decreases as the molecular cloud evolves. Figure 10 shows the map of $\mathrm{N}\left(\mathrm{N}_{2} \mathrm{H}^{+}\right) / \mathrm{N}\left(\mathrm{C}^{18} \mathrm{O}\right)$ relative abundance ratio. We can see that in the center of G341.244-00.265, the ratio is relatively high compared to the other parts of this filament. We regard the gas in the center of G341.244-00.265 as less evolved.

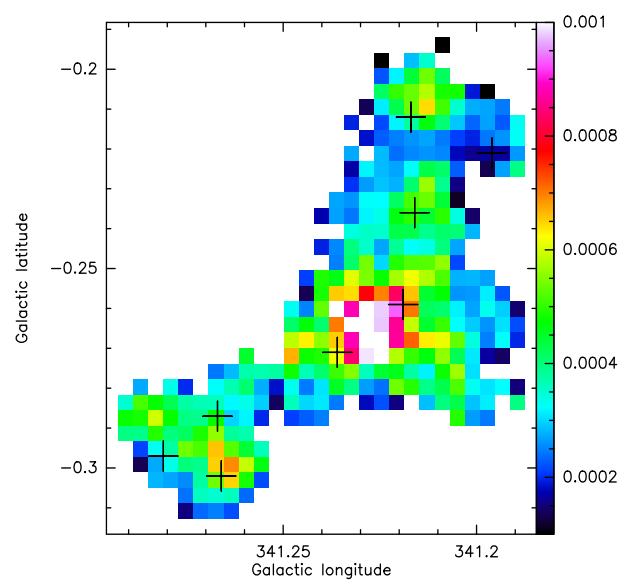

Fig. 10. $\mathrm{N}\left(\mathrm{N}_{2} \mathrm{H}^{+}\right) / \mathrm{N}\left(\mathrm{C}^{18} \mathrm{O}\right)$ relative abundance ratio map of G341.24400.265 . The plus signs indicate the dense clumps from Contreras et al. (2013).

\section{5. lonizing luminosity and ages of S22 and S24}

We used the SUMSS $834 \mathrm{MHz}$ radio continuum data to estimate the Lyman continuum fluxes and dynamical ages of S22 and S24. Assuming an electron temperature of $T_{e}=10^{4} \mathrm{~K}$, the number of UV ionizing photons needed to keep an HII region ionized is given by (Chaisson 1976; Guzmán et al. 2012)

$N_{\mathrm{L}}=7.6 \times 10^{46}\left(\frac{S_{v}}{\mathrm{Jy}}\right)\left(\frac{D}{\mathrm{kpc}}\right)^{2}\left(\frac{v}{\mathrm{GHz}}\right)^{0.1}\left(\frac{T_{e}}{10^{4} \mathrm{~K}}\right)^{-0.45} \mathrm{~s}^{-1}$,

where $v$ is the frequency and $S_{v}$ is the integrated flux density, and $D$ is the kinematic distance $(3.6 \mathrm{kpc})$ to the HII region. We derived $\mathrm{N}_{\mathrm{L}} \sim 9.3 \times 10^{47} \mathrm{ph} \mathrm{s}^{-1}$ and $9.0 \times 10^{47} \mathrm{ph} \mathrm{s}^{-1}$ for $\mathrm{S} 22$ and $\mathrm{S} 24$, respectively. Based on the ionizing fluxes for massive stars given by Martins et al. (2005), we estimated the spectral types of the ionizing stars of S22 and S24 to be $09.5 \mathrm{~V}$.

Assuming the two HII regions expand in a homogeneous medium, their dynamical ages could be estimated through (Dyson \& Williams 1980)

$t_{\mathrm{dyn}}=\frac{4 R_{\mathrm{s}}}{7 c_{\mathrm{s}}}\left[\left(\frac{R_{\mathrm{HII}}}{R_{\mathrm{s}}}\right)^{7 / 4}-1\right]$,

where $c_{\mathrm{S}}$ is the sound velocity in the ionized gas, assumed to be $10 \mathrm{~km} \mathrm{~s}^{-1} ; \mathrm{R}_{\mathrm{HII}}$ is the radius of the HII region; $R_{\mathrm{S}}$ is the original Strömgren radius given by $R_{\mathrm{S}}=\left(3 \mathrm{~N}_{\mathrm{L}} / 4 \pi n_{\mathrm{i}}^{2} \alpha_{B}\right)^{1 / 3}$, where $\mathrm{N}_{\mathrm{L}}$ is the ionizing luminosity calculated above; $n_{\mathrm{i}}$ is the initial $\mathrm{H}$ number density of the gas; and $\alpha_{B}=2.6 \times 10^{-13} \mathrm{~cm}^{3} \mathrm{~s}^{-1}$ is the hydrogen recombination coefficient. We found the dynamical ages are about $2.1 \times 10^{5}$ and $2.2 \times 10^{5}$ yr for S22 and S24, respectively.

\subsection{Distributions of YSOs}

We used the highly reliable GLIMPSE I catalog to search for YSOs in this filamentary cloud. The regions in the top panel of Fig. 11 indicate stellar evolutionary stages based on the criteria described by Allen et al. (2004): Class II sources are disk-dominated objects. These objects lie in the region $0<$ [3.6]$[4.5]<0.8$ and $0.4<[5.8]-[8.0]<1.1$, and their IR excess is caused by accretion disks around the YSOs. Class I sources are protostars with interstellar envelopes. Their locations in the color-color [3.6]-[4.5] vs. [5.8]-[8.0] diagram are delineated by 

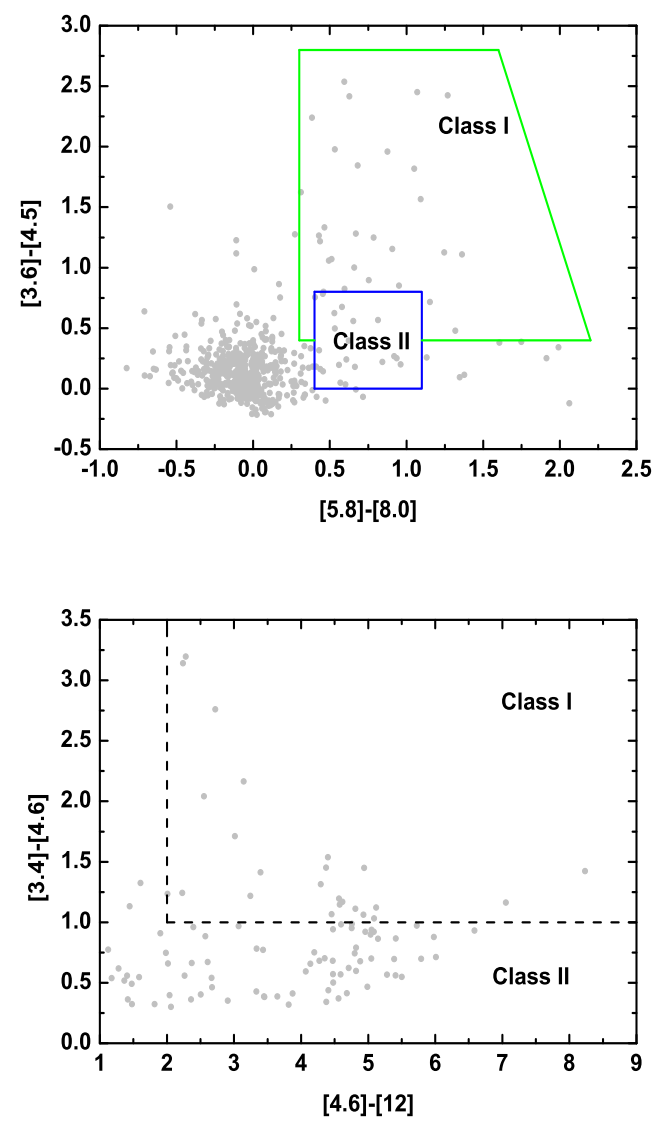

Fig. 11. Top panel: GLIMPSE [5.8]-[8.0] vs. [3.6]-[4.5] diagram for selected sources in the region of G345.244-00.265. Class I and Class II regions are indicated according to the criteria given by Allen et al. (2004). Bottom panel: YSO candidates from the WISE database.

the green lines in Fig. 11. We also used photometric data from the WISE survey to identify YSO candidates in this region. According to the criteria of Koenig et al. (2012), Class I candidates are selected if their colors match [3.4]-[4.6] $>1.0$ and [4.6][12.0] $>2.0$, while Class II candidates are selected with colors [3.4]-[4.6] $>0.25+\sigma([3.4]-[4.6])$ and [4.6]-[12.0] $>1.0+\sigma$ ([4.6]-[12.0]), where $\sigma(\ldots)$ indicates a combined error, added in quadrature. The locations of Class I and Class II candidates in color-color diagrams (CCDs) are shown in Fig. 11. The spatial distributions of the selected YSOs are shown in Fig. 12. We note that most of the YSOs distributed in the center of this filament are Class I sources, while most Class II candidates are located in the head and tail of G341.244-00.265, indicating star formation activities began on the two ends of this filament first.

\section{Discussions}

\subsection{Fragmentation}

The gravitational stability of a filament can be estimated by comparing its linear mass density $(M / l)$ with the virial linear mass density $\left((M / l)_{\mathrm{vir}} \equiv 2 \sigma_{v}{ }^{2} / \mathrm{G}\right)$. The value $\sigma_{v}$ is the one-dimensional total (thermal plus nonthermal) velocity dispersion of the average molecular gas. We derived the velocity dispersion from the average FWHM of $\mathrm{C}^{18} \mathrm{O}(2-1)$ line. The linear mass per unit length $(M / l)$ of G341.244-00.265 is about $1654 M_{\odot} \mathrm{pc}^{-1}$, while the virial linear mass density is only about $627 M_{\odot} \mathrm{pc}^{-1}$, indicating turbulence inside is unable to prevent the cloud from gravitational collapse. From the ATLASGAL catalog of

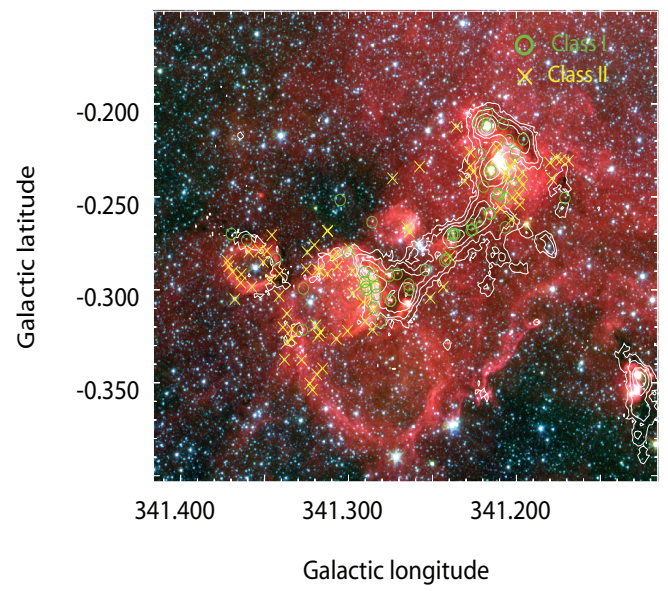

Fig. 12. Spacial distributions of YSOs on the three-color image created using the Spitzer 3.6 (blue), 4.5 (green), and 8.0 (red) $\mu \mathrm{m}$ IRAC band filters. Class I candidates are shown by green circles, while Class II candidates are shown by yellow crosses. The white contours are the same as shown in the top panel of Fig. 1.

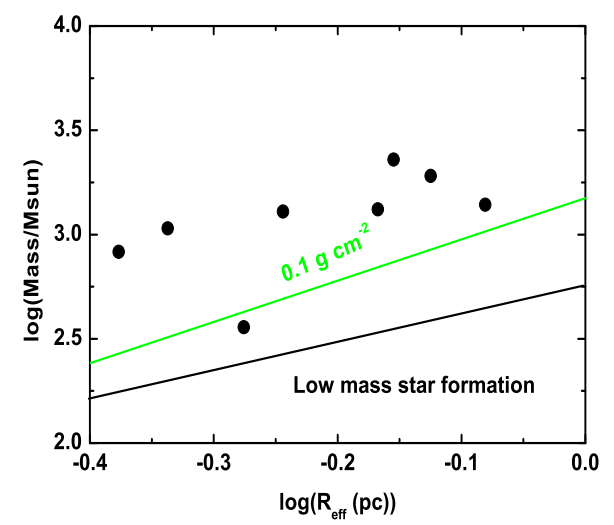

Fig. 13. Mass vs. radius plot of the eight clumps embedded in G341.24400.265 . The region below the black line represents the space devoid of massive star formation, where $M(r)<870 M_{\odot}(r / p c)^{1.33}$ (Kauffmann \& Pillai 2010).

Contreras et al. (2013), we found eight dense clumps involved in this region. Half of these (AGAL341.217-00.212, AGAL341.21900.259, AGAL341.236-00.271, and AGAL341.266-00.302) show infall motions (He et al. 2015), indicating star formation activities are ongoing actively in this filamentary cloud. We consider the mass-size relationship of these clumps to find out whether they have sufficient mass to form massive stars. The effective radius can be determined by $r=\sqrt{L_{\text {maj }} L_{\text {min }}}$. In this case, $L_{\text {maj }}$ and $L_{\min }$ are the deconvolved major and minor axes of each clump. According to Kauffmann \& Pillai (2010), the threshold for massive star formation is $M(r) \geq 870 M_{\odot}(r / p c)^{1.33}$. Figure 13 presents the mass versus radius plot of the eight clumps embedded in G341.244-00.265. We found all these dense clumps lie above the threshold, indicating they are candidates of massive star formation regions.

\subsection{Dynamic structure of the filament}

The dynamic structure can be a useful tool to study the formation and evolution of a filament. Velocity gradients perpendicular to the major axis have been postulated as effects of filament rotation (Olmi \& Testi 2002) and/or convergent flows (Schneider et al. 2010). On the other hand, velocity gradients 


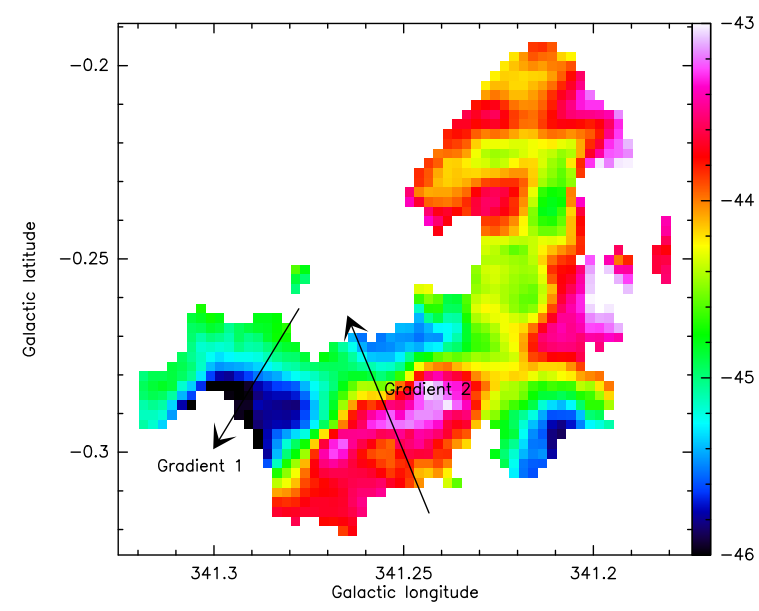

Fig. 14. Velocity-field (moment 1) map of ${ }^{13} \mathrm{CO}(2-1)$ of G341.24400.265 .

along filaments have been explained as mass inflowing toward the cloud cores (e.g., Kirk et al. 2013; Dhabal et al. 2018). Figure 14 presents the complex velocity-field (moment 1) map of G341.244-00.265 from the ${ }^{13} \mathrm{CO}$; the $\mathrm{N}_{2} \mathrm{H}^{+}$moment 1 map is not shown as they look very similar. At least two projected velocity gradients have been observed. The direction of velocity gradient 1 is toward S22 and has a value of about $0.85 \mathrm{~km} \mathrm{~s}^{-1} \mathrm{pc}^{-1}$. The CO molecular gas shows an arc-like structure around S22. The turbulent energy of the PDR around S22 could be estimated through $E_{\text {turb }}=\frac{1}{2} M \sigma_{3 \mathrm{~d}}^{2}$, where $\sigma_{3 \mathrm{~d}} \approx \sqrt{3} \sigma_{v}$, which is the threedimensional turbulent velocity dispersion. The derived turbulent energy of the PDR around S22 is about $1.2 \times 10^{47} \mathrm{erg}$. The ionization energy and thermal energy of S22 can be estimated as (Freyer et al. 2003)

$E_{\mathrm{i}}=\frac{4}{3} \pi n_{\mathrm{i}}\left(\frac{7}{4} c_{\mathrm{s}} R_{\mathrm{s}}^{5 / 2} t+R_{\mathrm{s}}^{7 / 2}\right)^{6 / 7} \chi_{0}$,

$E_{\mathrm{t}}=\frac{4}{3} \pi n_{\mathrm{i}}\left(\frac{7}{4} c_{\mathrm{s}} R_{\mathrm{s}}^{5 / 2} t+R_{\mathrm{s}}^{7 / 2}\right)^{6 / 7} \kappa T_{4}$,

where $\chi_{0}$ is the ionization potential $(13.6 \mathrm{eV})$ of hydrogen in the ground state. We found the ionization energy and thermal energy of S22 are about $5.1 \times 10^{47}$ erg and $0.3 \times 10^{47} \mathrm{erg}$, respectively. The ionization energy of S22 is 4 times larger than its surrounding PDR turbulent energy. Thus, we regard S22 as the likely driving source of velocity gradient 1 . The direction of velocity gradient 2 is from southwest to northeast and has a value of $0.62 \mathrm{~km} \mathrm{~s}^{-1} \mathrm{pc}^{-1}$. In Fig. 1, we can see that there seems to be an ionized shock in the southwest of G341.244-00.265. Bubble MWP1G341176-003905 may be the driving source for velocity gradient 2 . However, the SUMSS radio emission of MWP1G341176-003905 is very irregular. We could not estimate its ionization energy and thermal energy. If S22 and MWP1G341176-003905 are indeed the driving sources of the two velocity gradients, our study indicates that at least part of the large-scale dynamics in G341.244-00.265 originate from turbulence injections. The velocity gradients in the head are very complex. They cannot be explained by a single ration of the filament and/or shocks from bubble S24.

\subsection{Star formation scenario}

The distributions of YSOs suggest there is an age gradient of star formation in this filamentary cloud: most of the YSOs distributed in the center of this filament are Class I sources, while most Class II candidates are located in the head and tail of G341.24400.265 , indicating star formation activities began on the two ends of this filament first. The abundance ratio of $\mathrm{N}\left(\mathrm{N}_{2} \mathrm{H}^{+}\right) / \mathrm{N}\left(\mathrm{C}^{18} \mathrm{O}\right)$ is relatively higher in the center than that in the two ends of G341.244-00.265, also indicating the gas in the center is less evolved. Both observations and theories indicate expanding HII regions may trigger the next generation of star formation (e.g., Cichowolski et al. 2009; Miao et al. 2009; Panwar et al. 2014). Triggered star formation scenario indicates there should be an age gradient: the ages of stars decrease from the center to the outside of an expanding HII region. According to André \& Montmerle (1994), the age of Class I YSOs is $\sim 10^{5} \mathrm{yr}$, while Class II YSOs have a timescale of $\sim 10^{6} \mathrm{yr}$. However, the dynamical age of S22 and S24 is only about $2.2 \times 10^{5}$ yr. They are too young to trigger the star formation of the surrounding Class II YSOs. The study of Cappa et al. (2016) also indicates that bubble S24 is too young for triggering to have begun. Thus the age gradient could not be explained by the triggered star formation scenario. Numerical studies show that in a long but finite-sized filament, collapse may act a factor of two to three times faster at the ends of the filament than at its center (e.g., Pon et al. 2011, 2012), suggesting star formation at the ends of the filament prior to its center. Recently, Kainulainen et al. (2016) found the fragmentation strongly at the ends of the Musca cloud. Dewangan et al. (2017) also found massive clumps and YSO clusters prefer to locate at the both ends of the filamentary molecular cloud S242. These authors suggest the so-called end-dominated collapse may be responsible. Taking into account the distributions of YSOs and the $\mathrm{N}\left(\mathrm{N}_{2} \mathrm{H}^{+}\right) / \mathrm{N}\left(\mathrm{C}^{18} \mathrm{O}\right)$ ratio map, our study is also in agreement with the prediction of the so-called end-dominated collapse star formation scenario.

\section{Summary}

We performed a multiwavelength study toward molecular cloud G341.244-00.265 to investigate the physical and chemical properties, as well as the star formation activities taking place therein. The cloud shows an elongated filamentary structure both in farinfrared and molecular line emissions, with its head and tail associated with infrared bubbles S21, S22, and S24. G341.24400.265 has a linear mass density of about $1654 M_{\odot} \mathrm{pc}^{-1}$ and has a projected length of $11.1 \mathrm{pc}$. The cloud is prone to collapse based on the virial analysis. From the ATLASGAL catalog, we found eight dense massive clumps associated with this filamentary cloud. All of these clumps have sufficient mass to form massive stars. At least two velocity gradients have been found. S22 and MWP1G341176-003905 may be the driving source of the two velocity gradients. Using data from the GLIMPSE and WISE survey, we searched for YSO candidates in this region. We found an age gradient of star formation in this filamentary cloud: most of the YSOs distributed in the center are Class I sources, while most Class II candidates are located on the two ends of G341.244-00.265, indicating star formation at the two ends of this filament is prior to the center. The abundance ratio of $\mathrm{N}\left(\mathrm{N}_{2} \mathrm{H}^{+}\right) / \mathrm{N}\left(\mathrm{C}^{18} \mathrm{O}\right)$ is higher in the center than that in the two ends, also indicating the gas in the center is less evolved. Taking into account the distributions of YSOs and the $\mathrm{N}\left(\mathrm{N}_{2} \mathrm{H}^{+}\right) / \mathrm{N}\left(\mathrm{C}^{18} \mathrm{O}\right)$ ratio map, our study is in agreement with the prediction of the so-called end-dominated collapse star formation scenario.

Acknowledgements. We are very grateful to the anonymous referee for his/her helpful comments and suggestions. This paper has made use of information from the APEX Telescope Large Area Survey of the Galaxy. The ATLASGAL project 
is a collaboration between the Max-Planck-Gesellschaft, the European Southern Observatory (ESO) and the Universidad de Chile. This research made use of data products from the Millimeter Astronomy Legacy Team $90 \mathrm{GHz}$ (MALT90) survey. The Mopra telescope is part of the Australia Telescope and is funded by the Commonwealth of Australia for operation as National Facility managed by CSIRO. This paper is supported by National Natural Science Foundation of China under grants of 11503037.

\section{References}

Allen, L. E., Calvet, N., D’Alessio, P., et al. 2004, ApJS, 154, 363 André, P., \& Montmerle, T. 1994, ApJ, 420, 837

André, P., Men'shchikov, A., Bontemps, S., et al. 2010, A\&A, 518, L102

Battisti, A. J., \& Heyer, M. H. 2014, ApJ, 780, 173

Bergin, E. A., \& Langer, W. D. 1997, ApJ, 486, 316

Bergin, E. A., \& Tafalla, M. 2007, ARA\&A, 45, 339

Bergin, E. A., Ciardi, D. R., Lada, C. J., Alves, J., \& Lada, E. A. 2001, ApJ, 557, 209

Busquet, G., Estalella, R., Zhang, Q., et al. 2011, A\&A, 525, A141

Cappa, C. E., Duronea, N., Firpo, V., et al. 2016, A\&A, 585, 30

Chaisson, E. J. 1976, in Frontiers of Astrophysics, ed. E. H. Avrett (Harvard: Harvard University Press), 259

Chen, X., Shen, Z., Li, J., Xu, Y., \& He, J. 2010, ApJ, 710, 150

Cichowolski, S., Romero, G. A., Ortega, M. E., Cappa, C. E., \& Vasquez, J. 2009, MNRAS, 394, 900

Contreras, Y., Schuller, F., Urquhart, J. S., et al. 2013, A\&A, 549, A45

Contreras, Y., Rathborne, J. M., Guzman, A., et al. 2017, MNRAS, 466, 340

Cyganowski, C. J., Whitney, B. A., Holden, E., et al. 2008, AJ, 136, 2391

Cyganowski, C. J., Brogan, C. L., Hunter, T. R., Churchwell, E., \& Zhang, Q. 2011, ApJ, 729, 124

Deharveng, L., Schuller, F., Anderson, L. D., et al. 2010, A\&A, 523, A6

Dewangan, L. K., Baug, T., Ojha, D. K., et al. 2017, ApJ, 845, 34

Dhabal, A., Mundy, L. G., Rizzo, M. J., Storm, S., \& Teuben, P. 2018, ApJ, 853 , 169

Dislaire, V., Hily-Blant, P., Faure, A., et al. 2012, A\&A, 537, A20

Dyson, J. E., \& Williams, D. A. 1980, Physics of the Interstellar Medium (Manchester: Manchester University Press)

Enoch, M. L., Young, K. E., Glenn, J., et al. 2006, ApJ, 638, 293

Freyer, T., Hensler, G., \& Yorke, H. W. 2003, ApJ, 594, 888

Foster, J. B., Jackson, J. M., Barnes, P. J., et al. 2011, ApJS, 197, 25

Fuente, A., Martin-Pintado, J., Cernicharo, J., \& Bachiller, R. 1993, A\&A, 276 473
Guzmán, A., Garay, G., Brooks, K. J., \& Voronkov, M. A. 2012, ApJ, 753, 51 Guzmán, A., Sanhueza, P., Contreras, Y., et al. 2015, ApJ, 815, 130 He, Y. X., Zhou, J. J., Esimbek, J., et al. 2015, MNRAS, 450, 1926 Jackson, J. M., Rathborne, J. M., Foster, J. B., et al. 2013, PASA, 30, 57 Kainulainen, J., Hacar, A., Alves, J., et al. 2016, A\&A, 586, A27 Kauffmann, J., \& Pillai, T. 2010, ApJ, 723, L7 Keto, E., \& Rybicki, G. 2010, ApJ, 716, 1315

Kirk, H., Myers, P. C., Bourke, T. L., et al. 2013, ApJ, 766, 115 Klein, B., Hochgürtel, S., Krämer, I., et al. 2012, A\&A, 542, L3 Koenig, X. P., Leisawitz, D. T., Benford, D. J., et al. 2012, ApJ, 744, 130 Lada, C. J., Lombardi, M., \& Alves, J. F. 2010, ApJ, 724, 687 Ladd, N., Purcell, C., Wong, T., \& Robertson, S. 2005, PASA, 22, 62 Lee, J. E., Evans, N. J., II, Shirley, Y. L., \& Tatematsu, K. 2003, ApJ, 583, 789 Lee, J. E., Bergin, E. A., \& Evans, N. J. 2004, ApJ, 617, 360 Li, G.-X., Urquhart, J. S., Leurini, S., et al. 2016, A\&A, 591, A5 Martins, F., Schaerer, D., \& Hillier, D. J. 2005, A\&A, 436, 1049 Mauch, T., Murphy, T., Buttery, H. J., et al. 2003, MNRAS, 342, 1117 Miao, J., White, G. J., Thompson, M., \& Nelson, R. 2009, ApJ, 692, 382 Molinari, S., Swinyard, B., Bally, J., et al. 2010, A\&A, 518, L100 Moore, T. J. T., Bretherton, D. E., Fujiyoshi, T., et al. 2007, MNRAS, 379, 663 Olmi, L., \& Testi, L. 2002, A\&A, 392, 1053

Ossenkopf, V., \& Henning T. 1994, A\&A, 291, 943

Pagani, L., Daniel, F., \& Dubernet, M.-L. 2009, A\&A, 494, 719 Panwar, N., Chen, W. P., Pandey, A. K., et al. 2014, MNRAS, 443, 1614 Pon, A., Johnstone, D., \& Heitsch, F. 2011, ApJ, 740, 88 Pon, A., Toalá, J. A., Johnstone, D., et al. 2012, ApJ, 756, 145 Purcell, C. R., Longmore, S. N., Burton, M. G., et al. 2009, MNRAS, 394, 323 Ragan, S. E., Henning, T., Tackenberg, J., et al. 2014, A\&A, 568, A73 Rathborne, J. M., Whitaker, J. S., Jackson, J. M., et al. 2016, PASA, 33, e030 Schneider, N., Csengeri, T., Bontemps, S., et al. 2010, A\&A, 520, A49 Schuller, F., Menten, K. M., Contreras, Y., et al. 2009, A\&A, 504, 415 Schuller, F., Csengeri, T., Urquhart, J. S., et al. 2017, A\&A, 601, A124 Simpson, R. J., Povich, M. S., Kendrew, S., et al. 2012, MNRAS, 424, 2442 Siringo, G., Kreysa, E., Kovács, A., et al. 2009, A\&A, 497, 945

Tobin, J. J., Bergin, E. A., Hartmann, L., et al. 2013, ApJ, 765, 18 Vassilev, V., Meledin, D., Lapkin, I., et al. 2008, A\&A, 490, 1157 Vigren, E., Zhaunerchyk, V., Hamberg, M. et al. 2012, ApJ, 757, 34 Wang, K., Testi, L., Ginsburg, A., et al. 2015, MNRAS, 450, 4043 Whitworth, A. P., Bhattal, A. S., Chapman, S. J., Disney, M. J., \& Turner, J. A. 1994, MNRAS, 268, 291

Wilson, T. L., \& Rood, R. 1994, ARA\&A, 32, 191

Yu, N. P., \& Xu J. L. 2016, ApJ, 833, 248

Zinnecher, H., \& Yorke, H. W. 2007, ARA\&A, 45, 481 